\title{
Antibiotic resistance among Escherichia coli isolates from stool samples of children aged 3 to 14 years from Ujjain, India
}

\author{
Pragya Shakya ${ }^{1 \dagger}$, Peter Barrett ${ }^{2 \dagger}$, Vishal Diwan ${ }^{2,3^{*}+}$, Yogyata Marothi ${ }^{1}$, Harshada Shah $^{1}$, Neeraj Chhari ${ }^{4}$,
} Ashok J Tamhankar ${ }^{2,5}$, Ashish Pathak ${ }^{2,6,7}$ and Cecilia Stålsby Lundborg ${ }^{2}$

\begin{abstract}
Background: Antibiotic resistance is a major global public health concern, particularly in settings where few treatment options are available. Limited research has been done on antibiotic resistance in Escherichia coli of Indian children at community level. Therefore we studied antibiotic resistance patterns in E. coli isolates from stool samples of children aged 3-14 years from Ujjain, Central India, to investigate associations of resistance with demographic variables.
\end{abstract}

Methods: Children, 3-14 years of age, were included from 30 randomly selected villages of Palwa demographic surveillance site, Ujjain, India. Parents were interviewed using a questionnaire, and stool samples were collected from participating children. E. coli were isolated from stool samples $(n=529)$, and susceptibility testing to 18 different antibiotics was done using standard methods.

Results: The proportions of isolates resistant to various antibiotics were, nalidixic acid, (45\%), tetracycline (37\%), ampicillin (37\%), sulfamethoxazole/trimethoprim (29\%) and amoxicillin/clavulanic acid (29\%). No isolates were resistant to imipenem. Overall, $72 \%$ of isolates were resistant to at least one antibiotic and 33\% were multi-drug resistant. High rates of cross-resistance were seen for 15 (83\%) of the antibiotics studied. E. coli isolates from children with literate mothers were more resistant to penicillins and fluoroquinolones. ESBL-producers comprised 9\% of the isolates.

Conclusion: Antibiotic resistance and cross-resistance were common in E. coli from stools of children. Resistance rates were associated with maternal literacy.

Keywords: E. coli, Faecal, Children, Commensal, Antibiotic resistance, Asia

\section{Background}

Antibiotic resistance is a major global public health concern [1], particularly in settings where few treatment options are available, either due to lack of availability or affordability of second line therapies. Commensal Escherichia coli can act as reservoirs of resistance genes in the human gut. These resistant genes might be rapidly transferred to other commensal or pathogenic organisms [2,3]. Faecal E. coli is regarded as a useful indicator of the spread of acquired antibiotic resistance genes in the community $[4,5]$.

\footnotetext{
* Correspondence: vishaldiwan@hotmail.com

${ }^{\dagger}$ Equal contributors

${ }^{2}$ Global Health (IHCAR), Department of Public Health Sciences, Karolinska Institutet, Stockholm, Sweden

${ }^{3}$ Department of Public Health and Environment, R.D. Gardi Medical College, Ujjain, India

Full list of author information is available at the end of the article
}

Limited research has been done with regards to antibiotic resistance in E. coli among Indian children. In the few studies conducted, wide variation has been demonstrated in resistance rates of $E$. coli isolates from reportedly healthy children $[6,7]$. In the geographical area of the present study, high rate of broad-spectrum antibiotic prescribing for self-limiting conditions has been shown among children [8,9]. The high rate of antibiotic prescribing is likely to result in high rates of resistance. The main aim of this study was to describe prevalence of resistance in E. coli isolates from reportedly healthy children in the community, towards antibiotics commonly used locally. The secondary aim was to investigate associations of resistance with demographic variables.

\section{Methods}

This cross-sectional study was conducted in the Palwa demographic surveillance site (DSS) of Ruxmaniben

\section{Biomed Central}


Deepchand Gardi Medical College (RDGMC), Ujjain, Central India, between January and March 2011. The district of Ujjain has a population of almost two million people and $61 \%$ live in rural area [10]. The DSS contains a total of 60 villages, and has been described elsewhere [11]. Computer generated random numbers were used to select 30 villages from the DSS. The study design is summarised in Figure 1. Healthcare in Ujjain district is provided in public hospitals, charitable hospitals and private clinics. There are also large numbers of non-allopathic practitioners from traditional medical systems, and informal healthcare providers (IHP). Most qualified healthcare professionals work in the private sector in urban areas, whereas most of the IHP work in rural areas $[12,13]$.

Children aged between 3 - 14 years were included in the study. OpenEpi software was used to calculate a required sample size of 372 , given an anticipated frequency of resistance to at least one antibiotic of $50 \%$, with 95\% confidence interval [14]. The actual number of participants sought exceeded the sample size due to concerns of non-participation and to try to ensure a minimum of 10 stool samples obtained per village. One child per family was included in the study.

Trained research assistants visited selected children's homes. They discussed the study with the children's parents/guardians, invited them to participate and informed about the right to withdraw at any time and assured confidentiality. Parents who consented for their children to participate were included (Figure 1). Parents were interviewed using a structured questionnaire, which included questions on demographic details of the child and family, recent illness among the child and healthseeking behaviour in the event of illness. Research

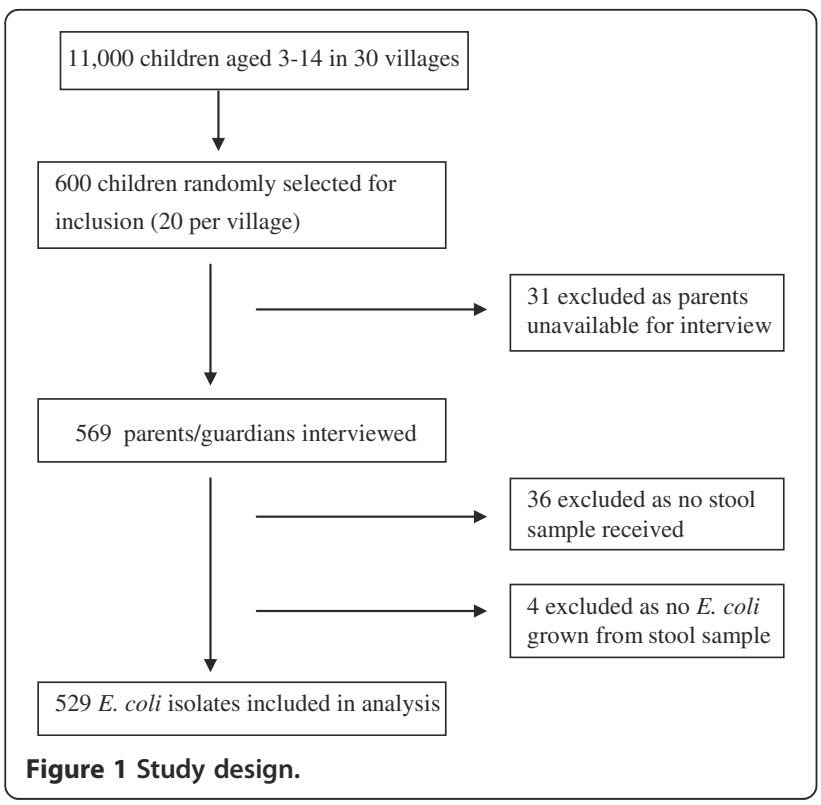

assistants provided sterile stool sample collection containers and collected the samples the following morning from each home.

All stool samples were transported to microbiology laboratory at RDGMC. Processing was started within four hours of sample collection. Stool samples were streaked on MacConkey agar for isolation of E. coli. Colonies morphologically resembling $E$. coli were confirmed by using biochemical identification tests [15]. E. coli isolate from each stool sample [16] was subjected to antimicrobial susceptibility testing using Kirby-Bauer disc diffusion method. CLSI interpretative criteria for susceptibility and resistance were used [17].

ATCC E. coli 25922 was simultaneously tested as control with each batch of antimicrobial susceptibility testing performed. After verifying the results of the standard strain, test sample results were interpreted. Two microbiologists read zone diameters independently by using antibiotic zone scale (Himedia, Mumbai, India).

Antibiotic susceptibility was tested for antibiotics commonly used in healthcare facilities and in the community in Ujjain district. Multi-drug resistance (MDR) was defined as resistance of an isolate to any antibiotic from at least three different antibiotic groups [18]. Extended-spectrum betalatamases (ESBLs) were phenotypically detected by the combined disc diffusion method with cefotaxime $(30 \mu \mathrm{g})$ and cefotaxime/clavulanic acid (30/10 $\mu \mathrm{g})$ and ceftazidime $(30 \mu \mathrm{g})$ and ceftazidime/clavulanic acid (30/10 $\mu \mathrm{g})$ [17]. Klebsiella pneumoniae ATCC 700603 was used as control for testing ESBL production.

Questionnaire responses and drug susceptibility data were collected, cleaned and entered in to IBM SPSS Statistics 20.0 (SPSS Inc., Chicago, IL, USA). Data were analysed using descriptive statistics, frequencies and bivariate analyses (cross-tabulations). A significance level of $p=0.05$ was used. Associations were determined between socio-demographic variables and health-seeking behaviour with the outcomes (i) resistance to one antibiotic, and (ii) MDR. Associations were first tested using chi-squared tests. Those variables which approached statistical significance $(\mathrm{p}<0.2)$ were entered in to multivariate logistic regression models with backward elimination. Age was used as a covariate. Independent variables used were: sex (male versus female), family type (joint versus nuclear), number of family members (up to five versus six or more), economic status (above poverty line versus below poverty line), maternal education (no education versus at least one year education), maternal occupation (exclusively homemaker versus any other work), paternal occupation (exclusively agriculture versus any other work), acute illness in child e.g., upper respiratory tract infections, diarrhea etc. (yes versus no), and recent antibiotic prescription or remaining unused antibiotic verified at household (yes versus no). 
The study was approved by the Ethics Committee of RDGMC, Ujjain, India (114/2010).

\section{Results}

Table 1 shows the demographic details of the families of the 529 children from whom isolates were obtained. The median age of the included children was 9 years. Thirty percent $(n=159)$ of them had been reported ill in the three weeks prior to the study. Among the children that were reported ill 26\% $(n=42)$ had received an antibiotic.

Table 1 Demographic details of participants and their families

\begin{tabular}{|c|c|c|c|}
\hline & & $N=$ & 529 \\
\hline & & $\mathbf{n}$ & (\%) \\
\hline Sex of children & Male & 304 & 58 \\
\hline & Female & 225 & 42 \\
\hline Family type $^{a}$ & Nuclear & 215 & 41 \\
\hline & Joint & 314 & 59 \\
\hline Economic status & Below poverty line ${ }^{b}$ & 226 & 44 \\
\hline & Above poverty line & 228 & 56 \\
\hline Caste $^{c}$ & Scheduled caste & 167 & 32 \\
\hline & Scheduled tribe & 4 & 1 \\
\hline & Other backward caste & 164 & 31 \\
\hline & Other & 194 & 36 \\
\hline Number of family members & Up to 5 members & 199 & 38 \\
\hline & 6 or more members & 330 & 62 \\
\hline Paternal education & Illiterate & 111 & 21 \\
\hline & 1-5 years education & 183 & 35 \\
\hline & $\geq 6$ years education & 235 & 44 \\
\hline Maternal education & Illiterate & 344 & 65 \\
\hline & 1-5 years education & 138 & 26 \\
\hline & $\geq 6$ years education & 47 & 9 \\
\hline Paternal occupation & Agricultural work & 328 & 62 \\
\hline & Labour work & 153 & 29 \\
\hline & Other work & 48 & 9 \\
\hline Maternal occupation & Housework only & 233 & 44 \\
\hline & Housework + Labour work & 147 & 28 \\
\hline & Housework + Agricultural work & 145 & 27 \\
\hline & Other work & 4 & 1 \\
\hline
\end{tabular}

${ }^{a}$ Nuclear family refers to only parents and children living in one household, i.e. a home sharing one kitchen. Joint family refers to parents and children and any other related or unrelated persons living in one household, i.e. a home sharing one kitchen.

${ }^{b}$ Those classified as living below the poverty line were those living in families in possession of a 'Below poverty line' ration card issued by the Government of India.

'Scheduled castes, backward castes and scheduled tribes are groups in society who were historically subject to social disadvantage and exclusion. They are awarded special status by the Constitution of India and receive certain social benefits. (Government of India. Ministry of Home Affairs. Office of the Registrar General \& Census Commissioner 2012).
Among the 529 E. coli isolates from 529 children, resistance to at least one antibiotic was observed in $72 \%$ of isolates $(\mathrm{n}=328)$. MDR was found in one third of isolates $(33 \%, \mathrm{n}=174)$. ESBL-producers comprised $9 \%(n=48)$ of isolates.

The rates of resistance observed for individual antibiotics, and selected combinations of antibiotics, are shown in Table 2. Table 3 summarises demographic variables, which were significantly associated with resistance to selected individual antibiotics. Isolates from children of mothers with at least one year of education were more resistant to norfloxacin [OR 2.3, 95\% CI $1.3-4.3$; p $<0.01$ ], ciprofloxacin [OR 2.8, 95\% CI 1.4 - 5.6; $\mathrm{p}<0.01$ ], piperacillin [OR 1.7, 95\% CI 1.1 - 2.5; $\mathrm{p}=0.013$ ], ampicillin [OR 1.6, 95\% CI $1.1-2.4 ; \mathrm{p}=0.017]$ and amoxicillin/clavulanic acid [OR 1.8, 95\% CI $1.2-2.7 ; \mathrm{p}<0.01$ ]. Paternal education was not associated with resistance in commensal $E$. coli of children. Isolates obtained from girls were more resistant to tetracycline compared to isolates from boys [OR 1.5, 95\% CI $1.1-2.2 ; \mathrm{p}=0.025$ ].

Prevalence of resistance to any antibiotic varied widely between different villages, from $40-100 \%$ of isolates, and

Table 2 Prevalence of resistance of $E$. coli to individual antibiotics and to combination of selected antibiotics from different groups

\begin{tabular}{|c|c|c|}
\hline \multirow[b]{2}{*}{ Antibiotic } & \multicolumn{2}{|c|}{$N=529$} \\
\hline & $n$ & $(\%)$ \\
\hline Tetracycline (A) & 197 & 37 \\
\hline Ampicillin (B) & 197 & 37 \\
\hline Piperacillin & 175 & 33 \\
\hline Amoxicillin/Clavulanic acid & 154 & 29 \\
\hline Piperacillin/Tazobactam & 12 & 2 \\
\hline Ciprofloxacin & 40 & 8 \\
\hline Norfloxacin & 57 & 11 \\
\hline Nalidixic acid (C) & 239 & 45 \\
\hline Cotrimoxazole (D) & 154 & 29 \\
\hline Cefoxitin & 22 & 4 \\
\hline Cefotaxime & 70 & 13 \\
\hline Ceftazidime & 72 & 14 \\
\hline Ceftriaxone (E) & 71 & 13 \\
\hline Cefepime & 71 & 13 \\
\hline Imipenem & 0 & 0 \\
\hline Gentamicin & 22 & 4 \\
\hline Amikacin & 26 & 5 \\
\hline Chloramphenicol & 23 & 4 \\
\hline$A+B$ & 110 & 21 \\
\hline$A+B+C$ & 72 & 14 \\
\hline$A+B+C+D$ & 62 & 12 \\
\hline$A+B+C+D+E$ & 25 & 5 \\
\hline
\end{tabular}


Table 3 Prevalence (\%) of resistance of $E$. coli to selected antibiotics associated with demographic variables

\begin{tabular}{|c|c|c|c|c|c|c|c|c|c|}
\hline & & TET & AMP & AMC & FEP & CIP & NOR & ADR & MDR \\
\hline \multirow[t]{2}{*}{ Sex of child } & Female & $43 *(* *)$ & 41 & 31 & $17 *$ & 9 & 12 & 75 & 36 \\
\hline & Male & 33 & 35 & 28 & 11 & 7 & 10 & 69 & 31 \\
\hline \multirow[t]{2}{*}{ Family type } & Joint & $39(* *)$ & 35 & 29 & 13 & 7 & 14 & 72 & 33 \\
\hline & Nuclear & 34 & 41 & 29 & 14 & 8 & 9 & 72 & 34 \\
\hline \multirow[t]{2}{*}{ Maternal education } & $>1$ year & 38 & $43(* *)$ & $35^{*}(* *)$ & 16 & $13^{*(* *)}$ & $16^{*(* *)}$ & 72 & 36 \\
\hline & 0 years & 36 & 34 & 26 & 12 & 5 & 8 & 72 & 31 \\
\hline \multirow[t]{2}{*}{ Given antibiotics (last three weeks) } & Yes & 50 & 43 & 38 & 19 & $12(* *)$ & 12 & 81 & 36 \\
\hline & No & 36 & 37 & 28 & 13 & 7 & 11 & 71 & 33 \\
\hline
\end{tabular}

Associations of antibiotic resistance Percentage (\%) prevalence of resistance shown for each antibiotic according to selected demographic factors.

( $\mathrm{TET}=$ tetracycline; $\mathrm{AMP}=$ ampicillin; $\mathrm{AMC}=$ amoxicillin/clavulanic acid; FEP = cefepime; $\mathrm{CIP}=$ ciprofloxacin; NOR = norfloxacin; $\mathrm{ADR}=$ resistance to any antibiotic; MDR = multi-drug resistance).

* Statistically significant by chi-squared analysis.

**Statistically significant by multivariate logistic regression analysis, adjusted for age.

prevalence of MDR also varied from 0 - 58\%. Children who had been ill within three weeks prior to the study had slightly higher rates of carriage of $E$. coli which were resistant to at least one antibiotic ( $74 \%$ vs. $71 \%)$, and also of MDR (38\% vs. $31 \%$ ), but the differences were not statistically significant. Children who had received an antibiotic within the previous three weeks had higher odds of carriage of $E$. coli, which were resistant to ciprofloxacin [OR 9.1, 95\% CI 1.6 - 50.6; p = 0.012].

Age was not found to be significantly associated with resistance to individual antibiotics or MDR. Other variables analysed in relation to antibiotic resistance were caste, economic status, number of family members, paternal and maternal occupation, and health-seeking behaviour in the event of child illness. None of these variables were significantly associated with resistance of $E$. coli isolates.

\section{Discussion}

\section{Resistance patterns in children in Ujjain district}

To our knowledge, this is the first community-based study, which describes the prevalence of antibiotic resistance among E. coli isolated from reportedly healthy children in Central India. The study shows high rates of resistance to individual antibiotics, with $E$. coli isolates from most children $(72 \%)$ resistant to at least one antibiotic. This differs from previous resistance rates seen in E. coli isolates from children from southern (63\%) and eastern India (38-68\%) $[6,7]$. Geographical variation in resistance patterns has been reported previously $[7,19]$.

High rates of antibiotic prescribing have been reported from hospitals in the same geographical area [19]. There are about 475 private pharmacies in Ujjain where clients can buy drugs. Antibiotics are often dispensed without any prescription, and often by those who lack formal qualifications as pharmacists [19]. Antibiotics are also prescribed or dispensed by non-allopathic practitioners and IHPs [8], although they are not authorised to do so.
E. coli isolates in the study showed less susceptibility to first line antibiotics, such as penicillins, nalidixic acid, cotrimoxazole and tetracycline, which tend to be more affordable and accessible to families. Cross-resistance was also common in the isolates, and prevalence of MDR exceeded that seen elsewhere in India [6,7].This may be indicative of an evolving resistance gene pool in commensal E. coli in India. The resistance patterns are unsettling as they relate to healthy children, whereas generally resistance rates of $E$. coli isolates tend to be higher for hospitalised children, where pathogenic E. coli often dominate [20]. A previous study by our research group in the same area among women attending antenatal clinics documented resistance to at least one antibiotic in $94 \%$ of commensal E. coli isolates. A total of 109 (15\%) isolates were ESBL producing and 35 isolates were MDR (35\%) [21].

\section{Factors affecting resistance patterns}

There has been uncertainty over the effect of age on antibiotic resistance $[6,7,22,23]$. There is also ambiguity regarding the role a child's sex plays in susceptibility to antibiotics. Some studies have found greater proportion of resistance in $E$. coli isolates from male children [7,23,24], whereas others demonstrated higher figures in E. coli isolates from females $[25,26]$.

An association between maternal education and increased antibiotic resistance was seen in the present study. This factor affected particular antibiotic groups, namely penicillins and fluoroquinolones. Those children whose mothers had attended school were more likely to carry E. coli resistant to these antibiotic groups. Maternal education has previously been regarded as a proxy indicator for socioeconomic status (SES) of a family [27]. It has been seen that children of higher SES are more likely to receive antibiotics [28] and previous consumption increases risk of antibiotic resistance [29]. Educated women tend to have a more autonomous role in society compared to illiterate 
women [30]. However, SES was not independently associated with resistance of $E$. coli in this study.

Our findings showed variation in resistance rates in E. coli of nearby villages, although there are no marked differences between these villages. Similar variation has been reported in Southern India previously [6]. Access to antibiotics may account for some of the inter-village variation. The majority of rural private pharmacies in Ujjain district are located along main roads [13], and those living within easier reach of these are likely to have higher rates of consumption.

Environmental factors may also play a role in the variation of resistance rates between villages. Most villages in the study area have no centralised water supply. Exposure to environmental contaminants may thus, vary with differences occurring locally in village water supply. Sources of environmental contamination with resistant bacteria include human effluent and farm run-off; these have been detected in community drinking water in low-income countries $[7,31]$. Hospital effluent has also been found to be a source of antibiotics and antibiotic resistance genes in Ujjain district $[32,33]$. The handling of water storage containers used in different villages may also play a role in occurrence of differing rates of resistance, as contamination of household water with coliform bacteria occurs mostly after collection from water sources [34].

This study adds to existing knowledge about resistance in commensal $E$. coli in an Indian community setting. The large number of antibiotics included in the study provides a comprehensive overview of resistance pattern in commensal E. coli. However, information was not obtained regarding diarrhoeagenic and non-diarrhoeagenic strains of $E$. coli so we cannot distinguish resistance prevalence between these strains. Only one isolate was tested for antibiotic susceptibility for each child, therefore some resistant isolates may have been missed and the true resistance rates may have been underestimated.

\section{Conclusions}

Resistant E. coli are highly prevalent in intestinal flora of reportedly healthy children in Ujjain district. ESBL detection was however comparatively low. The resistance rates in this study differ from those previously reported in other parts of the country. There is a need to enhance local and national research and surveillance efforts to monitor resistance trends of commensal $E$. coli in community.

\section{Consent}

Written informed consent was obtained from the patient's guardian/parent/next of kin for the publication of this study.

\section{Competing interests}

The authors declare that they have no competing interests.

\section{Authors' contributions}

VD and PS initiated the project. AJT, CSL, NC, AP, HS and YM participated in developing the concepts, the design and the planning of the study. VD coordinated the study. VD and NC carried out the fieldwork. PS, YM and HS planned and carried out the microbiological analysis. PB, VD, AP, AJT and CSL were involved in analysis and interpretation of data. PB and VD prepared first draft. AJT, CSL, VD, NC, YM, HS and AP revised the paper critically for substantial intellectual content. All authors have read and approved the final manuscript.

\section{Acknowledgements}

The authors wish to thank the village health workers and Dr. D.N. Paliwal who assisted with data collection, and all participating children and families; Professor Ragini Macaden and Salesh Chandran for advice on the microbiology part of the study. This work was supported by the Swedish Research Council (K2007-70X-20514-01-3) and Asia Link (348-2006-6633). P.S. received partial funding from the Indian Initiative for Management of Antibiotic Resistance. V. D. is a recipient of scholarships from Erasmus Mundus External Cooperation Window India Lot 15 and the Swedish Institute. A.P. received scholarships from Erasmus Mundus External Co-operation Window Lot 15 India and the Swedish Research School for Global Health for doctoral studies at Karolinska Institutet.

\section{Author details}

'Department of Microbiology, R.D. Gardi Medical College, Ujjain, India. ${ }^{2}$ Global Health (IHCAR), Department of Public Health Sciences, Karolinska Institutet, Stockholm, Sweden. ${ }^{3}$ Department of Public Health and Environment, R.D. Gardi Medical College, Ujjain, India. ${ }^{4}$ Department of Community Medicine, R.D. Gardi Medical College, Ujjain, India. ${ }^{5}$ Department of Environmental Medicine, R.D. Gardi Medical College, Indian Initiative for Management of Antibiotic Resistance, Ujjain, India. ${ }^{6}$ Department of Paediatrics, R.D. Gardi Medical College, Ujjain, India. ${ }^{7}$ Department of Women and Children's Health, International Maternal and Child Health Unit, Uppsala University, Uppsala, Sweden.

Received: 13 May 2013 Accepted: 18 September 2013 Published: 14 October 2013

\section{References}

1. Gootz T: The global problem of antibiotic resistance. Crit Rev Immunol 2010, 30(1):79-93.

2. Salyers AA, Gupta A, Wang Y: Human intestinal bacteria as reservoirs for antibiotic resistance genes. Trends Microbiol 2004, 12(9):412-416.

3. Blake DP, Hillman K, Fenlon DR, Low JC: Transfer of antibiotic resistance between commensal and pathogenic members of the Enterobacteriaceae under ileal conditions. J Appl Microbiol 2003, 95(3):428-436.

4. Lester SC, Del Pilar PM, Wang F, Perez Schael I, Jiang H, O'Brien TF: The carriage of Escherichia coli resistant to antimicrobial agents by healthy children in Boston, in Caracas, Venezuela, and in Qin Pu China. N Engl J Med 1990, 323(5):285-289.

5. Nys S, Okeke IN, Kariuki S, Dinant GJ, Driessen C, Stobberingh EE: Antibiotic resistance of faecal Escherichia coli from healthy volunteers from eight developing countries. J Antimicrob Chemother 2004, 54(5):952-955.

6. Seidman JC, Anitha KP, Kanungo R, Bourgeois AL, Coles CL: Risk factors for antibiotic-resistant E. coli in children in a rural area. Epidemiol Infect 2009, 137(6):879-888.

7. Sahoo KC, Tamhankar AJ, Sahoo S, Sahu PS, Klintz SR, Lundborg CS: Geographical variation in antibiotic-resistant Escherichia coli isolates from stool, cow-dung and drinking water. Int J Environ Res Public Health 2012, 9(3):746-759.

8. Pathak D, Pathak A, Marrone G, Diwan V, Lundborg CS: Adherence to treatment guidelines for acute diarrhoea in children up to 12 years in Ujjain, India-a cross-sectional prescription analysis. BMC Infect Dis 2011, 11:32.

9. Pathak A, Mahadik K, Dhaneria SP, Sharma A, Eriksson B, Lundborg CS: Antibiotic prescribing in outpatients: Hospital and seasonal variations in Ujjain India. Scand J Infect Dis 2011, 43(6-7):479-488.

10. Government of India. Provisional Population Totals: Madhya Pradesh. Ministry of Home Affairs. Office of the Registrar General \& Census Commissioner, India. Census of India. http://censusindia.gov.in/2011-prov-results/ prov_data_products_MP.html. 
11. Fochsen G, Deshpande K, Diwan V, Mishra A, Diwan VK, Thorson A: Health care seeking among individuals with cough and tuberculosis: a population-based study from rural India. Int J Tuberc Lung Dis 2006, 10(9):995-1000.

12. De Costa A, Diwan V: Where is the public health sector?' Public and private sector healthcare provision in Madhya Pradesh, India. Health Policy 2007, 84(2-3):269-276.

13. Sabde YD, Diwan V, Saraf VS, Mahadik VK, Diwan VK, De Costa A: Mapping private pharmacies and their characteristics in Ujjain district. Central India. BMC Health Serv Res 2011, 11:351.

14. Open Source Epidemiologic Statistics for Public Health: OpenEpi Version 2.3.1. Sample size for a proportion or descriptive study. http://www.openepi. com/v37/Menu/OE_Menu.htm.

15. Turgeon M: Introduction to Microbiology. In Linné \& Ringsrud's Clinical Laboratory Science. The Basics and Routine Techniques. 5th edition. Missouri, USA: Mosby; 2007.

16. Mathai E, Chandy K, Thomas K, Antoniswamy B, Joseph I, Mathai M, Sorensen T, Holloway $K$ : Antimicrobial resistance surveillance among commensal Escherichia coli in rural and urban areas in Southern India. Trop Med Int Health 2009, 13(1):41-45.

17. Clinical Laboratory Standards Institute: Performance Standards for Antimicrobial Susceptibility Testing: Twentieth informational supplement. CLSI document M100-S21. PA, USA: Wayne; 2011.

18. Magiorakos AP, Srinivasan A, Carey RB, Carmeli Y, Falagas ME, Giske CG, Harbarth S, Hindler JF, Kahlmeter G, Olsson-Liljequist B, et al: Multidrug-resistant, extensively drug-resistant and pandrug-resistant bacteria: an international expert proposal for interim standard definitions for acquired resistance. Clin Microbiol Infect 2012, 18(3):268-281.

19. Perez-Trallero E, Garcia-de-la-Fuente C, Garcia-Rey C, Baquero F, Aguilar L, Dal-Re R, Garcia-de-Lomas J: Geographical and ecological analysis of resistance, coresistance, and coupled resistance to antimicrobials in respiratory pathogenic bacteria in Spain. Antimicrob Agents Chemother 2005, 49(5):1965-1972.

20. Sahuquillo-Arce JM, Selva M, Perpinan H, Gobernado M, Armero C, Lopez-Quilez A, Gonzalez F, Vanaclocha H: Antimicrobial resistance in more than 100,000 Escherichia coli isolates according to culture site and patient age, gender, and location. Antimicrob Agents Chemother 2011, 55(3):1222-1228.

21. Pathak A, Chandran S, Mahadik K, Macaden R, Stålsby LC: Frequency and factors associated with carriage of multi-drug resistant commensal Escherichia coli among women attending antenatal clinics in central India. BMC Infect Dis 2013, 13:199-208.

22. Bartoloni A, Pallecchi L, Benedetti M, Fernandez C, Vallejos Y, Guzman E, Villagran AL, Mantella A, Lucchetti C, Bartalesi F, et al: Multidrug-resistant commensal Escherichia coli in children. Peru and Bolivia. Emerg Infect Dis 2006, 12(6):907-913.

23. Bartoloni A, Cutts F, Leoni S, Austin CC, Mantella A, Guglielmetti P, Roselli M, Salazar E, Paradisi F: Patterns of antimicrobial use and antimicrobial resistance among healthy children in Bolivia. Trop Med Int Health 1998, 3(2):116-123.

24. Bartoloni A, Bartalesi F, Mantella A, Dell'Amico E, Roselli M, Strohmeyer M, Barahona HG, Barron VP, Paradisi F, Rossolini GM: High prevalence of acquired antimicrobial resistance unrelated to heavy antimicrobial consumption. J Infect Dis 2004, 189(7):1291-1294.

25. Vatopoulos AC, Varvaresou E, Petridou E, Moustaki M, Kyriakopoulos M, Kapogiannis D, Sarafoglou S, Fretzagias A, Kalapothaki V: High rates of antibiotic resistance among normal fecal flora Escherichia coli isolates in children from Greece. Clin Microbiol Infect 1998, 4(10):563-569.

26. Zaoutis TE, Goyal M, Chu JH, Coffin SE, Bell LM, Nachamkin I, McGowan KL, Bilker WB, Lautenbach E: Risk factors for and outcomes of bloodstream infection caused by extended-spectrum beta-lactamase-producing Escherichia coli and Klebsiella species in children. Pediatrics 2005, 115(4):942-949.

27. Allin S, Stabile M: Socioeconomic status and child health: what is the role of health care, health conditions, injuries and maternal health? Health Econ Policy Law 2012, 7(2):227-242.

28. Kumar R, Indira K, Rizvi A, Rizvi T, Jeyaseelan L: Antibiotic prescribing practices in primary and secondary health care facilities in Uttar Pradesh India. J Clin Pharm Ther 2008, 33(6):625-634.

29. WHO: The evolving threat of antimicrobial resistance. Options for action. Geneva, Switzerland: World Health Organisation; 2012.

30. Bloom SS, Wypij D, Das Gupta M: Dimensions of women's autonomy and the influence on maternal health care utilization in a North Indian City. Demography 2001, 38(1):67-78.
31. Gaur A, Ramteke PW, Pathak SP, Bhattacherjee JW: Transferable antibiotic resistance among thermotolerant coliforms from rural drinking water in India. Epidemiol Infect 1992, 109(1):113-120.

32. Diwan V, Tamhankar AJ, Khandal RK, Sen S, Aggarwal M, Marothi Y, Iyer RV, Sundblad-Tonderski K, Stalsby-Lundborg C: Antibiotics and antibiotic-resistant bacteria in waters associated with a hospital in Ujjain. India. BMC Public Health 2010, 10:414

33. Diwan V, Tamhankar AJ, Aggarwal M, Sen S, Khandal RK, Sen S, Stalsby-Lundborg C: Detection of antibiotics in hospital effluents in India. Curr Sci 2009, 97(12):1753.

34. Wright J, Gundry S, Conroy R: Household drinking water in developing countries: a systematic review of microbiological contamination between source and point-of-use. Trop Med Int Health 2004, 9(1):106-117.

\section{doi:10.1186/1471-2334-13-477}

Cite this article as: Shakya et al:: Antibiotic resistance among Escherichia coli isolates from stool samples of children aged 3 to 14 years from Ujjain, India. BMC Infectious Diseases 2013 13:477.

\section{Submit your next manuscript to BioMed Central and take full advantage of:}

- Convenient online submission

- Thorough peer review

- No space constraints or color figure charges

- Immediate publication on acceptance

- Inclusion in PubMed, CAS, Scopus and Google Scholar

- Research which is freely available for redistribution

Submit your manuscript at www.biomedcentral.com/submit
C Biomed Central 